\title{
Author's reply: Stent-in-stent technique resolves late stent obstruction
}

\author{
Yoshio Haga ${ }^{1}$ \\ Received: 21 February 2020 / Accepted: 29 February 2020 / Published online: 18 March 2020 \\ (c) The International Gastric Cancer Association and The Japanese Gastric Cancer Association 2020
}

\section{To the Editor}

I appreciate Professors $\mathrm{Hu}$ and $\mathrm{Yu}$ for their interest in and pursuit of our manuscript entitled "Treatment Option of Endoscopic Stent Insertion or Gastrojejunostomy for Gastric Outlet Obstruction due to Gastric Cancer: A Propensity Score-Matched Analysis" [1]. Responses are provided herein to their inquiries.

Regarding the preoperative nutritional index, we measured the Prognostic Nutritional Index (PNI). PNI, calculated by combining serum albumin levels and the total circulating lymphocyte count, has been reported to reflect the nutritional and immunological status of patients with various types of cancers [2]. As shown in Table 1, PNI was significantly lower in the stent group than in the gastrojejunostomy group. Therefore, malnutrition was more common in the stent group than in the gastrojejunostomy group. Following propensity score matching (PSM), similar PNI were observed in the two groups, as shown in Table 5 .

Regarding late complications associated with the metallic stent, stent obstruction occurred in 16 patients (19\%) until death, as described in the Results section. However, the majority of patients $(94 \%, 15 / 16)$ recovered following the stent-in-stent technique. Therefore, stent obstruction does not indicate the termination of treatment.

Limited information is currently available on the treatment of gastric outlet obstruction due to gastric cancer. A randomized controlled trial may not be feasible due to the rarity of the underlying conditions. Most physicians now select endoscopic stent insertion for patients with poor physical conditions, while gastrojejunostomy may be more

This reply refers to the comment available online at https://doi. org/10.1007/s10120-020-01050-y.

Yoshio Haga

haga-yoshio@amakusa.jcho.go.jp

Department of Surgery, Japan Community Healthcare Organization Amakusa Central General Hospital, 101 Higashi-machi, Amakusa-shi 8630033, Japan suitable for patients with good physical conditions. Therefore, a large selection bias is inevitable in real-world data. PSM has potential as a practical method for analyzing comparative effectiveness between two treatments [3]. Our PSM analysis revealed that endoscopic stent placement resulted in less postoperative morbidity than and a similar food intake and equivalent survival times to gastrojejunostomy. However, the two patient groups were not the same, even after PSM, and, thus, more convincing evidence based on a randomized comparison is needed. Alternatively, similar findings from another large-scale survey, preferably outside of Japan, will contribute to reinforcing the generalizability of our data.

Funding None.

\section{Compliance with ethical standards}

Conflict of interest The author declares that he has no conflict of interest associated with this manuscript.

\section{Reference}

1. Haga Y, Hiki N, Kinoshita T, Ojima T, Nabeya Y, Kuwabara S, et al. Treatment option of endoscopic stent insertion or gastrojejunostomy for gastric outlet obstruction due to gastric cancer: a propensity score-matched analysis. Gastric Cancer. 2020. https:// doi.org/10.1007/s10120-020-01040-0.

2. Hu Y, Shen J, Liu R, Feng Z, Zhang C, Ling L, et al. Prognostic value of pretreatment prognostic nutritional index in non-small cell lung cancer: a systematic review and meta-analysis. Int J Biol Mark. 2018;33:372-8.

3. Yao XI, Wang X, Speicher PJ, Hwang ES, Cheng P, Harpole DH, et al. Reporting and guidelines in propensity score analysis: a systematic review of cancer and cancer surgical studies. J Natl Cancer Inst. 2017. https://doi.org/10.1093/jnci/djw323.

Publisher's Note Springer Nature remains neutral with regard to jurisdictional claims in published maps and institutional affiliations. 